\title{
Severe bilateral Pseudomonas keratitis exacerbated by prolonged contact lens wear
}



doi: $10.5694 / \mathrm{mjal} 3.00223$

\section{Clinical record}

A 28-year-old man with a 4-day history of bilateral ocular pain and a 2-day history of bilateral vision loss and purulent discharge was referred by a local hospital to our tertiary hospital. At the time of symptom onset, he had been wearing prescription monthly wear silicone hydrogel contact lenses (for correction of myopia) while swimming in lakes in Banlung in north-east Cambodia. The pain had worsened over 24 hours and the patient thought he had removed his contact lenses. He was prescribed topical neomycin $0.3 \%$ to use 2 -hourly and topical moxifloxacin $0.5 \%$ to use three times a day in both eyes, and advised to return to Australia for follow-up. He had no prior medical problems.

On examination, both contact lenses were still in situ and the patient had only light perception. There was conjunctival and ciliary injection, with peripheral thinning of both corneas, especially in the inferior areas (Figure, $A$ and $B$ ). Both corneas were completely white and opaque, owing to dense corneal infiltrations involving the whole cornea. Subtotal epithelial defects were seen with $1.5 \mathrm{~mm}$ hypopyon in both eyes

The contact lenses were removed immediately. Both eyes were then treated empirically with hourly topical cephazolin $5 \%$ and gentamicin $1.5 \%$, topical prednisolone acetate $1 \%$ six times a day and topical cyclopentolate $1 \%$ three times a day. Preservative-free lubricant (white soft paraffin [57.3\% w/w] plus liquid paraffin [42.5\% w/w] ointment) was administered four times a day. The


A: Right eye at presentation. B: Left eye at presentation. C: Right eye at Day 74. D: Left eye at Day 74. patient was also started on intravenous meropenem $2 \mathrm{~g}$ three times a day, oral ciprofloxacin $750 \mathrm{mg}$ twice a day, oral ascorbic acid $500 \mathrm{mg}$ twice a day and oral doxycycline $100 \mathrm{mg}$ once a day.

On Day 2, the organism grown from corneal scrapes from both eyes and the contact lenses was identified as Pseudomonas aeruginosa. The patient had lost his contact lens case and solution while in Cambodia. On Day 4, disc diffusion revealed that the organism was susceptible to ciprofloxacin rather than cephazolin. Topical cephazolin 5\% was replaced with topical ciprofloxacin $0.3 \%$.

From Day 20, lubricant was used hourly along with $0.3 \%$ tobramycin ointment twice a day. Bilateral temporal tarsorrhaphy was performed on Day 20 to promote healing. The patient was started on oral prednisolone therapy on Day 22 to help reduce inflammation associated with the bulging and melting of the corneas. Topical antibiotic therapy was tapered down as he began to respond well.

On Day 53, the tarsorrhaphy was opened as the epithelium had completely healed. Scars involving almost the whole surface areas of both corneas remained. On Day 74, there was extensive scarring and neovascularisation of both corneas (Figure, $\mathrm{C}$ and D). On Day 97 , the patient's unaided visual acuity was counting fingers in the right eye at $75 \mathrm{~cm}$ and counting fingers in the left eye at $50 \mathrm{~cm}$, which improved with pinhole testing to $6 / 24-1$ and $6 / 36$, respectively.
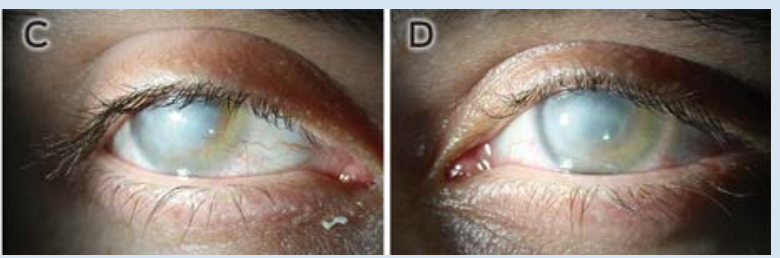

seudomonas aeruginosa is a gram-negative bacterium that is ubiquitous in the environment. It is found particularly in tropical areas, so lakes and rainforests in north-east Cambodia would be ideal places to come in contact with it. ${ }^{1-3}$

$P$. aeruginosa is usually susceptible to aminoglycosides and fluoroquinolones. ${ }^{4,5}$ Ofloxacin eye drops are effective in over $90 \%$ of bacterial contact lens-associated infections, are relatively non-toxic and do not need to be manufactured in a pharmacy, so could be used as first-line therapy for suspected microbial keratitis in contact lens wearers. ${ }^{2,4}$

Contact lens wear has led to a large rise in cases of Pseudomonas keratitis, ${ }^{6}$ owing to adherence of the bacterium to contact lens materials such as silicone hydrogel and the bacterium's resistance to disinfectants. ${ }^{1}$ Also, the quite static environment on the posterior side of a contact lens enables organisms to stay on the ocular surface for a long period and be protected from host defence mechanisms, and thereby replicate more easily. ${ }^{1,7}$

It is commonly believed that for microbial keratitis to occur there needs to be an injury to the cornea so that the organism can pass through the epithelium and into the corneal stroma. ${ }^{6,8}$ However, studies in rats have found that $P$. aeruginosa forms biofilms on the posterior surface of contact lenses and subsequently causes severe microbial keratitis without prior injury to the corneal epithelium. ${ }^{1}$ Endophthalmitis is also a risk if the organisms are present in sufficient quantities, as they can penetrate intact epithelium and endothelium. ${ }^{1,7}$ Risk factor analysis has found that microbial keratitis disease load is decreased by $60 \%-70 \%$ by avoiding night-time wear of lenses. ${ }^{9}$ Other research suggests a 20 times increased risk of microbial keratitis for patients who wear contact lenses while sleeping compared with those who wear daily disposable contact lenses. ${ }^{2,4}$ Our 


\section{Lessons from practice}

- In any contact lens wearer who presents with a sore eye, first check whether the contact lenses have been removed and remove them immediately if still in situ.

- In suspected Pseudomonas keratitis, fluoroquinolones and aminoglycoside topical antibiotics are generally very effective.

- Removing contact lenses before swimming in lakes, especially in tropical climates, is strongly advised.

- Daily wear disposable contact lenses have been shown to be associated with lower rates of microbial keratitis, and would be ideal for people who wear contact lenses while travelling.

patient had worn his lenses for at least 4 nights from when his symptoms began. The release of metalloproteinases from the P. aeruginosa is likely to have caused the rapid progression of the infection and subsequent corneal melting.

Bilateral Pseudomonas keratitis is rare, but this case shows how wearing contact lenses in a high-risk area and poor hygiene can lead to very severe bilateral microbial keratitis. Daily wear disposable lenses have been found to be the safest contact lenses, with the lowest rates of associated microbial keratitis, as compared with daily wear lenses that are put in solution overnight. ${ }^{2,4}$

After over 3 months of intense therapy, our patient is able to count fingers at less than 1 metre. He will almost certainly require corneal graft transplantation (which has its own risks) in 6-9 months for any chance to go back to his baseline functioning. We strongly advise that contact lenses be removed before swimming in lakes and that they be removed immediately if the wearer develops a sore eye.

Competing interests: No relevant disclosures.

Provenance: Not commissioned; externally peer reviewed.

1 Evans D, Fleiszig S. Why does the healthy cornea resist Pseudomonas aeruginosa infection? Am J Ophthalmol 2013; 155: 961-970.

2 Green M, Apel A, Stapleton F. Risk factors and causative organisms in microbial keratitis. Cornea 2008; 27: 22-27.

3 Parmar P, Salman A, Kalavathy CM, et al. Bilateral Pseudomonas keratitis without predisposing factors. Indian J Ophthalmol 2007; 55: 62-63.

4 Green M, Apel A, Stapleton F. A longitudinal study of trends in keratitis in Australia. Cornea 2008; 27: 33-39.

5 Hue B, Doat M, Renard G, et al. Severe keratitis caused by Pseudomonas aeruginosa successfully treated with ceftazidime associated with acetazolamide. J Ophthalmol 2009; 2009: 794935.

6 Marquart M, O'Callaghan R. Infectious keratitis: secreted bacterial proteins that mediate corneal damage. J Ophthalmol 2013; 2013: 369094.

7 Park JH, Song NH, Koh JW. Achromobacterxylosoxidans keratitis after contact lens usage. Korean J Ophthalmol 2012; 26: 49-53.

8 Li YC, Zeldovich A, Chua BJ, et al. Hazardous contact: a case of visual loss following Pseudomonas keratitis from novelty contact lens wear. Med J Aust 2006; 185: 173-174

9 Stapleton F, Carnt N. Contact lens-related microbial keratitis: how have epidemiology and genetics helped us with pathogenesis and prophylaxis. Eye 2012; 26: 185-193. 